\title{
Defect Distribution along Single GaN Nanowhiskers
}

\author{
Anna Cavallini, ${ }^{\dagger}$ Laura Polenta, ${ }^{, \dagger}$ Marco Rossi, ${ }^{\dagger}$ Thomas Richter, ${ }^{\ddagger}$ Michel Marso, ${ }^{\ddagger}$ \\ Ralph Meijers, ${ }^{\ddagger}$ Raffaella Calarco, ${ }^{\ddagger}$ and Hans Liuth ${ }^{\ddagger}$
}

Physics Department, University of Bologna, Viale Berti Pichat 6/2, 40127 Bologna, Italy, and Institute of Thin Films and Interfaces (ISG1) and

cni - Centre of Nanoelectronic Systems for Information Technology,

Research Center Jülich, 52425 Jülich, Germany

Received February 13, 2006

\begin{abstract}
In this letter we report on spectral photoconductivity (PC) on different sections of single MBE-grown GaN nanowhiskers of diameters ranging on the order of $100 \mathrm{~nm}$. The photoconductivity spectra show, besides the band-gap related transition, deep-levels corresponding to the yellow, green, and blue bands. A strong spatial localization of specific photocurrent peaks has been observed, indicating that the defects responsible for such transitions are distributed inhomogeneously along the column growth direction.
\end{abstract}

Gallium nitride nanostructures are currently an intense object of investigation in view of possible applications in nanoelectronics and nanolasing. ${ }^{1-4}$ Their properties have been considered suitable for photonic and biological nanodevices such as blue light emitting diodes, short wavelength ultraviolet nanolasers, and nanofluidic biochemical sensors, as well as for nanowire transistors. ${ }^{2,4-6}$

In recent years, the scientific community has been investigating GaN nanocolumns by electrical, structural, and optical characterization, ${ }^{2-7}$ focusing its effort on determining how the physical properties of the material behave at the nanoscale level.

For GaN nanocolumns, prepared in a bottom-up approach by MBE or MOCVD growth, a high level of size and density control has been achieved. However, knowledge of the defects contained in these structures and their spatial distribution is still lacking.

Recent results evidenced a strong correlation between column size and photoinduced electrical properties: the nanowire diameter affects the UV photoconductivity as well as the presence of persistent photoconductivity effects. ${ }^{4}$ Persistent photoconductivity (PPC) means that after removing the light excitation the photoinduced conductivity enhancement is observed to last for a long time. This phenomenon, which is, of course, of major importance for optoelectronics applications of $\mathrm{GaN}$, seems to reduce drastically with the nanostructure size.

The PPC effect is often observed to coexist with the yellow band luminescence in bulk GaN with a few micrometers

* Corresponding author. E-mail: laura.polenta@unibo.it; tel: ++39051 2095806; fax: ++390512095153.

University of Bologna.

$\doteqdot$ Research Center Jülich. thickness. Hence, it was suggested by some authors that the same defects could be responsible for both features. ${ }^{8-9}$ The yellow band is a widely known defect-related feature detected by both luminescence and photoconductivity techniques. Centered around 2.2-2.3 eV below the conduction band, it is almost omnipresent in gallium nitride and some indications about its surface-related nature have been reported. ${ }^{10-12}$ Nanowires have a high surface-to-volume ratio. Therefore, a detailed study of their defects is very fruitful to get a deeper insight into these new materials as well as to shed light on the character of the usually detected PC bands.

The GaN nanowires have been grown by radio frequency plasma-assisted MBE on Si (111) substrates. ${ }^{4}$ The whisker density and diameter, ranging between 20 and $500 \mathrm{~nm}$, can be controlled by monitoring the III/V ratio. N-rich growth conditions give rise to the columnar structure. ${ }^{3,4,13}$

The nanowires were released from the native silicon substrate by exposure to an ultrasonic bath and deposited on a Si (100) host substrate covered with an insulation layer of $\mathrm{SiO}_{2}(300 \mathrm{~nm})$ in order to perform the electrical conduction experiments. Ohmic contacts are patterned by electron beam lithography of $\mathrm{Ti} / \mathrm{Au}$.

Nanowhiskers with diameters on the order of $100 \mathrm{~nm}$ have been analyzed, and a typical sample is shown in Figure 1. Bottom and top parts of the nanowires have been unfailingly recognized thanks to the tapering effect present in the top part of sample 3. Two electrodes have been prepared at the ends and one approximately in the center. This three-contact configuration allows for distinguishing the signal contributions from the bottom part, containing interface-related defects, from those of the top part of the nanostructure. 


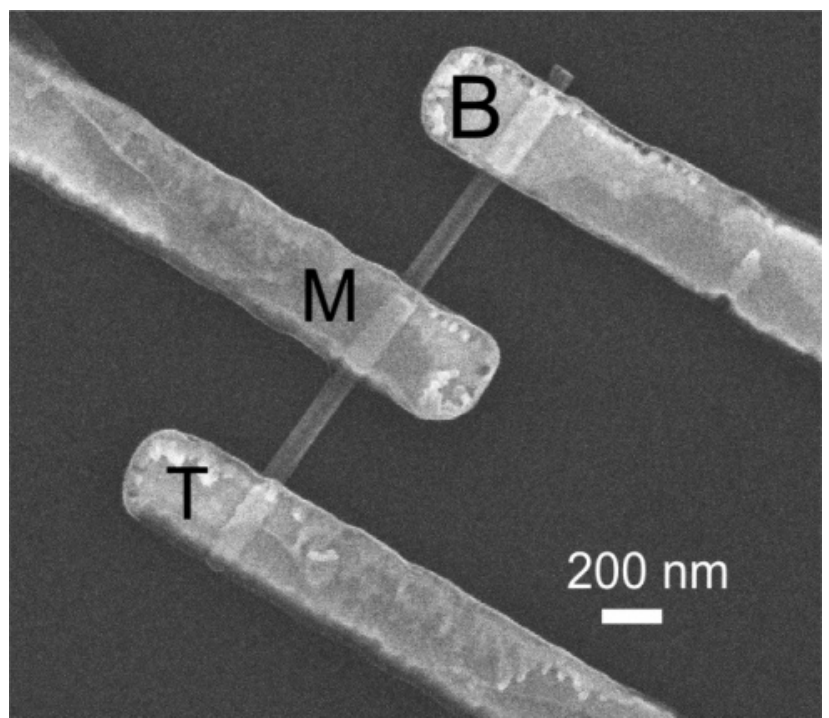

Figure 1. Typical $\mathrm{GaN}$ nanowisker deposited on the $\mathrm{SiO}_{2}(300$ $\mathrm{nm}) / \mathrm{Si}$ substrate with three ohmic contacts patterned by electronbeam lithography of Ti/Au. In this SEM (scanning electron microscopy) image obtained at $20 \mathrm{keV}$ of accelerating voltage, electrode T is located at the top, electrode B is located at base, and electrode $\mathrm{M}$ is approximately in the middle.

The nanowire shown in Figure 1 has a diameter of approximately $85 \mathrm{~nm}$. Three Ti/Au electrodes have been prepared on this wire: B (bottom), M (middle), and T (top). Electrode $\mathrm{B}$ is located at the base of the nanowire, previously attached to the silicon substrate, electrode $\mathrm{T}$ is near to the top facet, and electrode $\mathrm{M}$ is approximately at the middle of the wire.

Spectral photoconductivity has proven to be a very powerful tool in order to distinguish defect states responsible for optically induced transitions of carriers from defectrelated bands to the conduction or valence bands in wide gap materials. ${ }^{14-16}$ Depending on the excitation wavelength, the carriers can gain enough energy to jump from a deep level to their respective bands, respectively, from valence to conduction band. Because the photocurrent is an image of the electrical conductivity, all transitions in the valence or conduction bands are observed, independent of their radiative or nonradiative character.

All photocurrent measurements are performed at a constant electric field, that is, the bias voltage is adjusted to account for different electrode spacings.

Light emitted by a Xe lamp enters a monochromator and the monochromatic beam exiting through the output slit is focused onto the sample, modulated with a chopper frequency of $3-5 \mathrm{~Hz}$.

The photon flux ranges between $5 \times 10^{13}$ and $5 \times 10^{14}$ photons $/ \mathrm{cm}^{2} \mathrm{~s}$ depending on the wavelength. The photocurrent signal is collected by a lock-in amplifier. While the spectral range of wavelengths from 330 up to $650 \mathrm{~nm}$ is covered, the variation of current signal due to the release of photogenerated carriers from deep traps evidences deep center-to-band transitions.

Photocurrent spectra have been subsequently normalized to the photon flux in order to quantitatively define the amplitude of the bands with respect to the band-gap related transition.

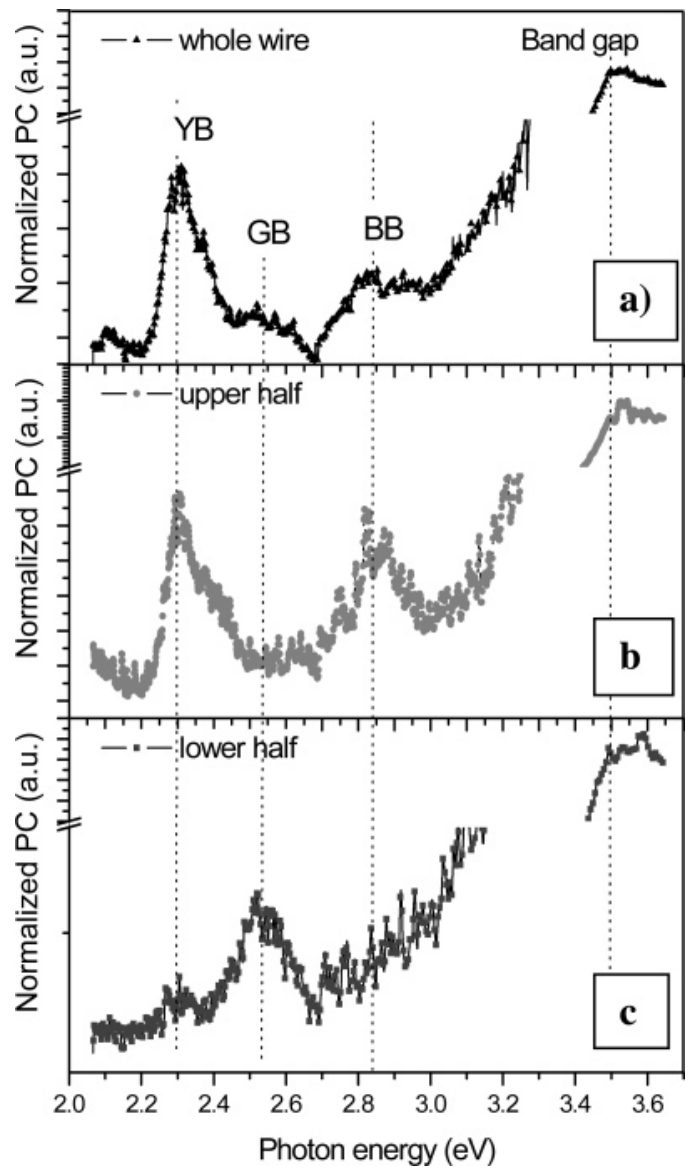

Figure 2. Photocurrent spectra of the sections of the nanowisker. PC spectra on a 85-nm-thick nanowire referring to (a) whole nanowire (electrodes $\mathrm{T}-\mathrm{B}$ ); (b) the upper half (electrodes $\mathrm{T}-\mathrm{M}$ ), and (c) the lower half (electrodes $\mathrm{B}-\mathrm{M}$ ) with respect to the growth direction. The $y$ axis is interrupted to account for the different orders of magnitude between near-band edge and defect-related band signals.

Figure 2a reports the $\mathrm{PC}$ spectrum relevant to the nanowire shown in Figure 1. Besides the band-gap related transition, other bands in the visible range are clearly distinguished at around 2.30, 2.52, and $2.85 \mathrm{eV}$.

These peaks correspond to the widely known luminescence bands denoted by yellow (YB), green (GB), and blue (BB) bands, which are usually observed in compact layers. ${ }^{10}$ Recent studies, based on photoluminescence and surface photovoltage spectroscopy, assign the green and yellow bands to complex defects involving gallium vacancies, $\mathrm{V}_{\mathrm{Ga}}$, which are related to bulk or surface states, respectively..$^{10-12}$ Photoconductivity measurements on different compact HVPE (hydride vapor phase epitaxy)-grown samples support the surface-related character of the yellow band. ${ }^{14}$

Although photoconductivity, because of its high energetic resolution, usually distinguishes the yellow and green transitions, luminescence detects a broad yellow band and is barely able to distinguish the green emission.

Photoluminescence (PL) spectroscopy carried out on the as-grown nanowiskers ensemble, before removal of the whiskers from the substrate, shows a broad yellow emission band (results not shown here). However, it is worth noting that it refers to the wide distribution of differently sized nanowires. The difficult detection of the green band could be 
related to a lower radiative efficiency or to the difference in the physical processes involved in these techniques. PL spectra are usually obtained by illuminating the samples with above-band-gap light to permit all of the carrier transitions giving rise to photon emission (radiative). Because at that wavelength the light is essentially absorbed by the surface, the signal is mostly sensitive to defects in the close proximity of the surface, which can mask weaker bulk-related transitions. Moreover, because the excitation beam in our PL setup is perpendicular to the sample surface and thus parallel to the whisker growth direction, it mainly excites the top part of the structures.

However, photoconductivity consists of a selective activation of the absorption processes that generate the transitions from the deep levels to the bands. The wavelength is varied to span all of the energies within the gap. Thus, the in-depth investigation is guaranteed for below band-gap energy. Furthermore, the light beam used in our photocurrent measurements is transverse to the nanocolumn and can penetrate the whole sample easily even for near band-gap energies. Spectral photoconductivity does select responses coming from different located volumes of the nanowires, just by conveniently choosing the electrodes: in this way the active volume contributing to the signal can be straightforwardly identified, differently from other techniques, such as micro-PL, whose spatial resolution is of the order of the sample size.

The local investigation on the two sections of the nanowires might thus give deeper insight into the origin of the photocurrent bands and their link with defects referring to interface, bulk, or surface.

The two spectra arising from the top part $(\mathrm{T}-\mathrm{M})$ and from the bottom part $(\mathrm{B}-\mathrm{M})$ of the nanowire shown in Figure 1 are shown in Figure $2 b$ and $c$. The main difference consists of the presence and in the amplitude of the green and yellow bands. The spectrum shown in Figure 2b, belonging to the upper half, contains the same bands as the whole nanowire (Figure 2a) but with different contributions. In particular, the green band signal is reduced significantly in Figure $2 b$. In contrast, the $\mathrm{B}-\mathrm{M}$ related spectrum (Figure $2 \mathrm{c}$ ), corresponding to the lower half of the nanowire, is characterized by a dominant green band.

Previous PC experiments carried out on the cross section of HVPE-grown compact layers, hence exciting the interface region directly, have shown the existence of a broad dominant blue-green band in the spectrum. ${ }^{17}$

In addition, we systematically observe a degradation of the signal-to-noise ratio in spectra carried out in the lower parts of the nanowires; it also occurs in thin HVPE-grown compact layers. ${ }^{18}$ This fact can be explained by taking into account the influence of interface-related defects on the current transport characteristics. Because of the thermal and lattice mismatch between $\mathrm{GaN}$ and substrate, a degenerate region at the interface is formed, where the presence of a dense distribution of line and point defects has been observed already. ${ }^{19}$ The increase of noise in our PC spectra can thus be assigned to the lower quality of the structure close to the interface GaN/Si(111). For the above-mentioned reason, it is hard to define the amplitude of the blue band in this
Table 1. Defect-to-Band-Edge Peaks Ratio Percentage Referring to the Spectra Shown in Figure $2^{a}$

\begin{tabular}{lccc}
\hline & $\begin{array}{c}\mathrm{YB} / \mathrm{BE} \\
(\%)\end{array}$ & $\begin{array}{c}\mathrm{GB} / \mathrm{BE} \\
(\%)\end{array}$ & $\begin{array}{c}\mathrm{BB} / \mathrm{BE} \\
(\%)\end{array}$ \\
\hline whole wire & 0.18 & 0.09 & 0.12 \\
upper half & 0.15 & 0.06 & 0.12 \\
lower half & 0.18 & 0.50 &
\end{tabular}

${ }^{a} \mathrm{BE}$ means band edge, and $\mathrm{YB}, \mathrm{GB}$, and $\mathrm{BB}$ refer to yellow, green, and blue bands.

spectrum, which might be masked by the rapid rise of the signal toward band-gap energies.

Although the density of defects is not achievable straightforwardly from these measurements, because of the many unknown parameters, we can however estimate their "impact" by analyzing the defect-to-band-gap ratio in each spectrum: the results are shown in Table 1.

It is evident from Table 1 that the yellow band amplitude is only slightly affected by the position along the column, whereas the green band signal increases by approximately 1 order of magnitude.

With a similar approach, we can quantitatively measure the relative weight between yellow and green bands in all of the nanowires analyzed, observing that the yellow/green ratio ranges between 2 and 5 in the upper half and between 0.1 and 0.4 in the lower half.

We can definitely assess that the green band transition is strongly localized in the lower part of the nanowire and is thus mainly related to defects originating at the interface with the silicon substrate. Alternatively, the yellow band, whose contribution is distributed homogeneously along the nanocolumn, is the dominant feature in the upper part, in agreement with its presumed surface-related character.

The spectrum referring to the whole wire reflects an average behavior, which takes into account both interfaceand surface-related contributions.

In conclusion, we have determined an inhomogeneous distribution of defects along the nanocolumn. The photoconductivity analysis on GaN nanowiskers shows how the green band is dominant in the first steps of the heteroepitaxial growth, hence related to defects originating at the interface to the substrate. By moving away from the interface, the green band contribution decreases and the yellow band feature dominates the spectrum.

Acknowledgment. This research was carried out in the framework of the VIGONI program (www.crui.it) funded by MIUR (Ministero Istruzione Universita' e Ricerca) and DAAD (Deutscher Akademischer Austauschdienst).

\section{References}

(1) Kuykendall, T.; Pauzauskie, P.; Zhang, Y.; Goldberger, J.; Sirbuly, D.; Denlinger, J. Yang, P. Nat. Mater. 2004, 3, 524-528.

(2) Gradeèak, S.; Qian, F.; Li, Y.; Park, H-G.; Lieber, C. M. Appl. Phys. Lett. 2005, 87, 173111.

(3) Meijers, R.; Richter, T.; Calarco, R.; Stoica, T.; Bochem, H.-P.; Marso, M.; Lueth, H. J. Cryst. Growth, in press; available online doi: 10.1016/j.jcrysgro.2005.11.117

(4) Calarco, R.; Marso, M.; Richter, T.; Aykanat, A. A.; Meijers, R.; Hart, A.v.d.; Stoica, T.; Lueth, H. Nano Lett. 2005, 5, 981-984 
(5) Huang, Y.; Duan, X.; Cui, Y.; Lieber, C. M. Nano Lett. 2002, 2, $101-104$

(6) Johnson, J.; Choi, H.; Yang, P.; Saykally, R. Nat. Mater. 2002, 1, 106.

(7) Dai, L.; Liu, S. F.; You, L. P.; Zhang, J. C.; Qin, G. G. J. Phys.: Condens. Matter 2005, 17, L445-L449.

(8) Reddy, C. V.; Balakrishnan, K.; Okomura, H.; Yoshida, S. Appl. Phys. Lett. 1998, 73, 224-226.

(9) Chen, H. M.; Chen, Y. F.; Lee, M. C.; Feng, M. S. J. Appl. Phys. 1997, 82, 899-901.

(10) Reshchikov, M. A.; Morkoc, H. J. Appl. Phys. 2005, 97, 061301

(11) Shalish, I.; Kronik, L.; Segal, G.; Rosenwaks, Y.; Shapira, Y.; Tisch, U.; Salzman, J. Phys. Rev. B 1999, 59, 9748-9751.

(12) Reshchikov, M. A.; Morkoc, H.; Park, S. S.; Lee, K. Y. Appl. Phys. Lett. 2002, 81, 4970-4972.
(13) Calleja, E.; Sánchez-Garcia, M.; Sánchez, F.; Calle, F.; Naranjo, F.; Muñoz, E.; Jahn, U.; Ploog, K. Phys. Rev. B 2000, 62, 16826.

(14) Castaldini, A.; Cavallini, A.; Polenta, L. Appl. Phys. Lett. 2005, 87, 122105

(15) Bube, R. H. Photoconductivity of Solids; Wiley: New York, 1960.

(16) Qiu, C. H.; Hoggatt, C.; Melton, W.; Leksono, M. W.; Pankove, J. I. Appl. Phys. Lett. 1995, 66, 2712-2714.

(17) Castaldini, A.; Cavallini, A.; Polenta, L. Phys. Status Solidi A 2005, 202, 2912-2919.

(18) Castaldini, A.; Cavallini, A.; Polenta, L. Appl. Phys. Lett. 2004, 84, 4851-4853

(19) Look, D. C.; Reynolds, D. C.; Hemsky, J. W.; Sizelove, J. R.; Jones, R. L.; Molnar, R. J. Phys. Rev. Lett. 2004, 79, 2273.

NL060332N 